\title{
MODELING AND CALCULATION OF AUTORESONANCE MECHATRONIC VIBRATION DEVICE
}

\author{
Vitaly Krupenin \\ Mechanical Engineering Research Institute of the Russian Academy of Sciences, Russia \\ krupeninster@gmail.com
}

\begin{abstract}
The paper deals with the problems of modeling vibration and vibro-impact autoresonant machines. Among the modern vibro-impact machines and devices operating in agricultural and industrial production, in construction and mining industry, a special place is occupied by autoresonance systems. These machines are the most productive and energy efficient. The principle of autoresonance lies in the fact that the designed technical objects work by realizing the resonant movements of the working bodies under the action of forces generated by the movement itself. Modeling such devices is often associated with the use of modern nonlinear mechanics, which in many cases uses various modifications of the energy balance principle. Since vibratory machines and devices in most cases implement periodic modes, this principle is reduced to fulfillment of the condition of balance of work of the forces of external energy sources and dissipation forces during the period of motion. The methods and capabilities of modern mechatronics allow the creation of autoresonance systems that allow balancing of the work of the forces and also even the forces themselves. To do this, you need to use special electromechanical devices that allow you to create the necessary compensating feedback. The present paper briefly describes the theory of such systems and then considers an example of calculating a device that allows to reproduce the necessary periodic impacts. We consider the device dynamic models with arbitrary dynamic compliance operators. Analysis is made of the organization of autoresonance technological machines of vibroimpact action, based on the creation of a feedback circuit, including the measurement of impulses of the working organ, which is the determining parameter of motion in the corresponding conservative model of the technological process. Examples and calculation formulas are given. It is indicated that similar principles of organization of machines can be constructed in the measurement of other determining parameters of motion.
\end{abstract}

Keywords: vibro-impact system, autoresonance, dynamic compliance, energy balance, compensation.

\section{Introduction}

Vibration machines and devices are widely distributed and are used in agricultural and industrial production, mining, construction, and in many other industries. Among vibration machines, as a rule, machines of vibro-impact action are distinguished by the highest efficiency, since the technological processes they implement are associated with systematic shocks of solids [1-4].

Obtaining stable and predictable results of processing workpieces or media with such machines is possible only under the condition that they implement the most effective resonant processes under conditions of constantly changing technological load.

It turns out that these difficulties can be avoided, if you create a self-oscillating excitation system. Such a system is implemented by introducing a special feedback loop, which generates an exciting force through a non-linear transformation of the signal proportional to certain parameters of the movement of the working body (tool, impacter, etc.).

When tuning, which is called autoresonance, with any changes in the parameters of the oscillating system and technological load, the resonant state is automatically maintained in the system [5].

Let the system establish a periodic process. During the period of tool movement, the work of the excitation and dissipation forces must be balanced. That is, there should be a balance of works of nonconservative forces. In this paper, we consider the problem of modeling the device, which at each moment of time provides the balance of the forces themselves, that is, the dissipation forces are compensated not on average, but uniformly [6].

\section{General view of the system}

Consider the scheme of the vibro-impact device of a fairly general form. Using the methods described in [2;7], we represent the object under study as a linear system with an arbitrary number of degrees of freedom (Fig. 1) containing a working body (impacter, etc.) and control subsystem. The impacter is modeled by a point of mass $m$. Since the workflow is vibro-impact, the system presented is 
strongly non-linear. Additional nonlinearities determine the feedback elements included in the control subsystem.

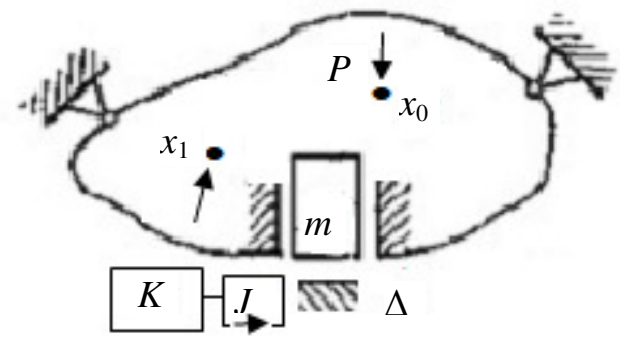

Fig. 1. General view of studied mechatronic vibro-impact system

Suppose that as a result of constructing a correct mathematical model or as a result of carrying out full-scale measurements a system of operators of dynamic compliance is specified $[7 ; 8]$. Such a system completely describes the linear part of the object under consideration. The working process consists in the organization of periodic collisions between the impacter and the fixed obstacle, for example, the workpiece surface.

Denote the $x$ coordinate of the impacter with the mass $m$. Suppose that the impacter performs onedimensional oscillations along a certain axis. Value $\Delta>0$ - there is a gap. The case $\Delta<0$ corresponds to preload. The preload can be provided by the system design as well as in the case, if at the point $x_{0}$, a constant force $P$ is applied, which presses the working member to the surface to be treated.

At point $x_{1}$, a control force $K$ is applied, the form of which we are going to determine based on the characteristics of the auto-oscillatory system. This force is obviously formed by organization of the feedback loop. Near the point of contact of the impacter with the treated surface a sensor is placed that measures some parameters of its movement, for example, the impact impulse $J$. The control action is formed in accordance with the sensor signal.

We will designate the operators of dynamic compliance, which correspond to the force acting at the point $x_{n}$ of moving the point $x_{k}$, as $L\left(x_{n}, x_{k} ; p\right) ; p$ - an operator of differentiation. Given the scheme given in Fig. 1, for the localization point of the workflow $x$ you can write the operator equation

$$
x(t)=L\left(x_{0}, x ; p\right) P+L\left(x_{1}, x ; p\right) K-L(x, x ; p) \Phi(x, \dot{x}),
$$

where the expression $\Phi(x, \dot{x})$ denotes the force of the impact interaction $[9 ; 10]$. We will assume that the impact is modeled using the Newton hypothesis, that is, it is momentary, and we also assume that it is elastic.

Energy losses in the implementation of the workflow can be considered, for example, by introducing the corresponding components into the representation for the operator [2;5], as well as by using special techniques for modeling impact using more realistic hypotheses [7].

Suppose, finally, that all converters and sensors included in the system are inertia-free.

In the absence of friction and control action, the equation of motion of the conservative system corresponding to (1) takes the form:

$$
x(t)=L\left(x_{0}, x ; 0\right) P-L(x, x ; p) \Phi(x, \dot{x}),
$$

where it is considered that the force $P=$ const. And, since here we neglect friction, it is assumed that for all operators $L(x, x ; p): \operatorname{Im}[L(x, x ; i \omega)]=0$.

Let $u \equiv L\left(x_{0}, x ; 0\right) P$. Periodic mode with one impact interaction for the period of movement of the conservative system $\left(T_{0}\right)$ under the assumption that the origin of time coincides with the impact can be represented as [7]:

$$
x(t)=u-J \chi\left(t, \omega_{0}\right) ; \omega_{0}=2 \pi T_{0}^{-1},
$$

where the periodic Green function (PFG) corresponding to the operator $L(x, x ; p)$ is given by the Fourier series of the form: 


$$
\chi\left(t, \omega_{0}\right)=\sum_{k=-\infty}^{\infty} L\left(x, x ; i k \omega_{0}\right) \exp \left(i k \omega_{0} t\right) .
$$

The impact impulse in this case of an elastic impact will be given by the ratio $J=2 m|\dot{x}(-0)| \geq 0$ and based on the impact condition. From here it is easy to get:

$$
J=\frac{[u-\Delta]}{\chi\left(0, \omega_{0}\right)} .
$$

In a conservative vibroimpact system, the impact impulse turns out to be the integral of motion mutually uniquely associated with the total energy $E$.

The considered auto-oscillatory system will be organized in such a way that the feedback should be constructed as a result of measuring the values of any of the integrals of motion, for example, the shock impulse. It will be shown that the result will be a convenient way for organization of autoresonance vibration-impact machines.

The frequency ranges for the existence of the solution (3), (5) are determined in specific cases from the condition $x(t) \leq \Delta$. In practice, the condition $J \geq 0$ is checked, which is equivalent in most cases. Relation (5) defines the equation of the skeleton curve $\left(J=J\left(\omega_{0}\right)\right)$. then:

We rewrite the equation of motion (1) using the dynamic stiffness operator $L^{-1}(p)$ [4;7]. We have

$$
L^{-1}(x, x ; p) x=P_{x}+F(p, x)-\Phi(x, \dot{x}) .
$$

Here it is designated: $P_{x}=L^{-1}(x, x ; 0) L\left(x_{0}, x ; p\right) P ; \quad F(p, x)=L^{-1}(x, x ; 0) L\left(x_{1}, x ; p\right) K$.

We will form the control action $F(p, x)$ in such a way that in the initial system (1) it is possible to implement periodic self-oscillations, which would retain the shape of the motion mode of the conservative system (3).

Let the dynamic stiffness operator be represented as follows: $L^{-1}(x, x ; p)=W_{1}(x, x ; p)+W_{2}(x, x ; p)$, and $\operatorname{Im} W_{1}(x, x ; p)=\operatorname{Re} W_{2}(x, x ; p)$. Solutions (3), (5) were constructed under the assumption that $W_{2}(x, x ; p) \equiv 0$. We substitute the representation (3) into the relation (6) at a certain value of frequency $\omega_{0}$ satisfying the condition $x(t) \leq \Delta$ and we find as a result

$$
W_{2}(x, x ; p)\left[u-J \chi\left(t, \omega_{0}\right)\right]=F\left[p, u-J \chi\left(t, \omega_{0}\right)\right] .
$$

We will further look for the function $F(p, x)$ in the subclass of functions with the structure $\{\beta(J) W(x, x ; p)\}$, where a certain function $\beta(J)$ is differentiable on any finite interval, and $W(x, x ; p)$ is a meromorphic function [7] of a complex variable $p$. Taking into account that $W_{2}(x, x ; p) u=0$, from the relation (7) we find:

$$
W_{2}(x, x ; p)\left[J \chi\left(t, \omega_{0}\right)\right]=\beta(J) W(x, x ; p)\left[J \chi\left(t, \omega_{0}\right)\right] .
$$

Therefore, it is necessary to fulfill the conditions

$$
W_{2}(x, x ; p)=W(x, x ; p), \beta(J)=1 .
$$

Thus, the conditions determining the structure of the control force $F(p, x)$ are obtained. From the second relation (8), one can find the stationary values of the pulse $J^{0}$, and from the inversion of the skeletal curve $J(\omega)$, the frequencies of the self-oscillations.

The choice of function $\beta(J)$, along with the structure of the operator of the linear part of the system, determines the nature and efficiency of the control system. From what follows, it will be possible to see that it is very convenient to choose a monotonically decreasing function.

Let us choose, for definiteness, $\beta(J)=\beta_{0} J^{1}, \beta_{0}=$ const $>0$. Then the stationary value of the impact impulse $J^{0}=\beta_{0}$ and the frequency of self-oscillations is determined from the relation $\beta_{0}=J(\omega)$ Since the value of the integral of motion (impact pulse) lies exactly on the skeletal curve corresponding to the realization of nonlinear resonances, the conditions found ensure the realization of autoresonance motions of the type described. 


\section{Sustainability research}

The found solutions need to be investigated for sustainability. In engineering calculations, the socalled energy condition is often used $[2 ; 3 ; 5]$. Strictly speaking, the energy condition is necessary. The efficiency of using this condition in applied problems is widely known [2-5].

We write the function $E(J)$ that corresponds to the balance of work of non-conservative forces for the period of movement $T$ :

$$
E(J)=-J^{2} \int_{0}^{T}\left[W_{2}(x, x ; p) \chi(t, \omega)-\beta(J) W_{2}(x, x ; p) \chi(t, \omega)\right] p \chi\left(t, \omega_{0}\right) d t
$$

Here, the first term on the right side corresponds to the work of the dissipation forces, and the second to the work of the forces of an external energy source.

After some transformations we get:

$$
E(J)=-J^{2} \lambda[1-\beta(J)] ; \lambda=\text { const }>0
$$

For asymptotically stable periodic regimes with a stationary value of the integral of motion (in this case, an impulse of impact), the dissipation forces stabilize the system and therefore in accordance with the energy condition

$$
\frac{d E(J)}{d J}<0\left(\text { with } J=J^{0}\right)
$$

Given the calculations done, we get

$$
E_{J} \equiv \frac{d E(J)}{d J}=J^{2} \lambda \frac{d \beta}{d J} .
$$

At $E_{J}<0$, self-oscillations are asymptotically stable. By choosing the function - monotonously decreasing, we ensured the stability of the system. In the case, when $\beta(J)=\beta_{0} J^{1}, \beta_{0}=$ const $>0$ : $E_{J}=-\beta_{0} \lambda<0$. Thus, in accordance with the energy condition, the stationary mode of motion $J^{0}=\beta_{0}$ is asymptotically stable. More precisely stability analysis can be performed using other modern methods [9-12].

From the technical point of view, organization of such a regime allows, with a minimum of energy costs, to achieve maximum efficiency of the technological process. Questions about the features of practical implementation of the corresponding device are an independent problem and are not discussed in this article. We can specify several ways to solve it. Important recommendations can be found in [9].

In conclusion of the theoretical part of the paper, we note that the notion of autoresonance of the vibration system was introduced in the book [13]. This concept has proven to be very useful in practical engineering. The principles of autoresonance are used in the design of many vibration, vibroimpact and ultrasonic machines and devices $[3-5 ; 8 ; 9 ; 16]$. These principles underlie the special types of cyclic robots [14;15;17], as well as many other important technical objects.

\section{An example of a system with one degree of freedom}

As an interesting example, consider a system with one degree of freedom [6;7] (Fig. 2).

The linear oscillator vibrates, impacting with a fixed obstacle 2, on which the impact impulse sensor is installed, which is forming a signal proportional to the magnitude $J$. This signal is converted to some function $\beta(J)$. After multiplying with the signal coming from the speed sensor 1 , the result is transmitted to the exciter 4. Assuming the impacter mass is equal to one, we write the equation of motion in the operator form

$$
\left(p^{2}+\Omega^{2}+2 b p\right) x=-\Phi(x, \dot{x})+\beta(J) p x,
$$

where $b>0-$ coefficient of viscous friction. Because a system with a gap is considered here, the force $P=0$. In the conservative case ( $b=\beta(J)=0)$, solutions (3), (5) have the form [7]:

$$
x(t)=-J \chi(t) ; \chi(t)=\left[2 \Omega \sin 1 / 2 \Omega \mathrm{T}_{0}\right]^{-1} \cos \Omega\left(t-1 / 2 \mathrm{~T}_{0}\right) ; J=-2 \Omega \operatorname{tg}\left(\pi \Omega \omega_{0}^{-1}\right) .
$$


The representation for the periodic Green function $\chi(t)$ in the second formula (12) takes place only for $t \in[0, T]$. Generally speaking, for all $t \in(-\infty, \infty)$ it is necessary to use the Fourier series (4), when $L\left(x, x ; i k \omega_{0}\right)=\left(\Omega^{2}-k^{2} \omega_{0}^{2}\right)^{-1}$. The set of natural frequencies of the conservative vibro-impact system is $\Omega \leq \omega<2 \Omega$.

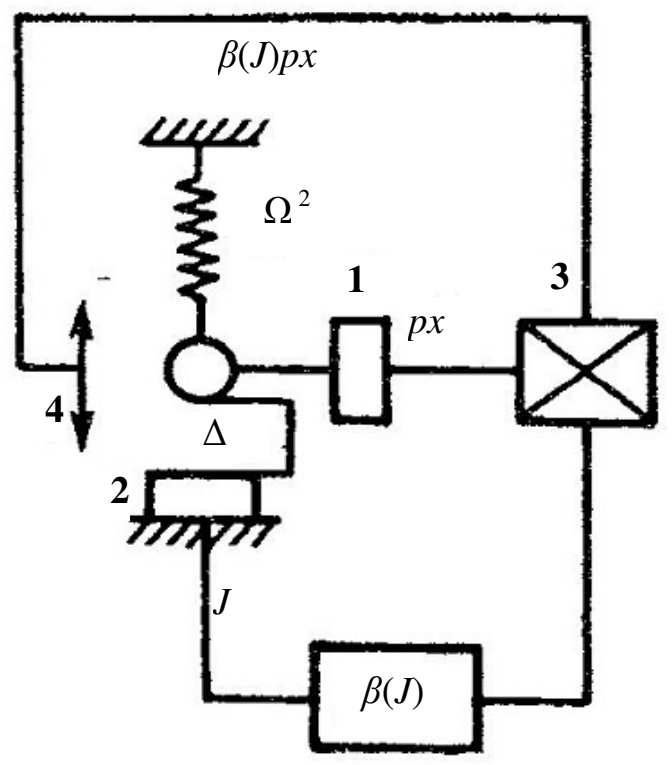

Fig. 2. Example of system with one degree of freedom:

1 - speed sensor; 2 - impact impulses sensor; 3 - signal multiplier; 4 - vibration exciter

In this case $W_{1}(p)=p+\Omega^{2}, W_{2}(p)=2 b p$. When $\beta(J)=\beta_{0} J^{-1}$ the stationary value of the impact impulse turns out to be $J^{0}=0.5 \beta_{0} b^{-1}$.

The solution has the form described by the first formula (12). From the third formula (12) we find the frequency of self-oscillations

$$
\omega=\frac{\pi \Omega}{\pi-\operatorname{arctg}\left[\beta_{0} /(4 b \Omega \Delta)\right]}
$$

In accordance with the energy condition (10), the found mode is asymptotically stable. More accurate studies that can be performed numerically or using modern methods of nonlinear mechanics [12] confirm this conclusion.

\section{Results and discussion}

The main result of the article is to describe a specific class of autoresonance devices with a remarkable property: the impacter performs the movement characteristic of the conservative system. Thus, the considered systems can be attributed to the class of systems with full compensation of friction forces at each moment of time.

\section{Conclusions}

1. It should be noted that the organization of the machines in question requires, obviously, to provide in the design a device intended for a rigid start of the vibro-impact mode of movement $[4 ; 8]$, since at least one blow must occur for organizing the measurement and fixation of shock impulses. Devices for the rigid start usually give the impacter additional energy, for example, the system is shaken.

2. For systems with a large number of degrees of freedom organization of an autoresonance scheme of the type in question may require the use of other integrals of motion.

3. Accounting for the loss of energy during an impact using the recovery coefficient hypothesis, generally speaking, takes beyond the scope of this class of devices, since the rebound of the impacter simulated according to Newton cannot be accurately described using a conservative model. This circumstance is of a model nature and does not fundamentally affect the physical 
properties of the systems under consideration. Accounting for energy losses during an impact can be performed using an "equivalent correction" in the viscous friction coefficient: $b=b_{1}+r \pi^{-1} \Omega$, where $b_{1}$, is the "true" viscous friction coefficient, $r=1-R, R$ is the coefficient of recovery speed at impact $(0<R \leq 1)$.

\section{Acknowledgements}

This work was supported by the Russian Science Foundation under the grant No 19-19-00065.

\section{References}

[1] Вибрации в технике: Справочник. Т.4. Вибрационные машины и процессы. (Vibration in Engineering: Handbook. Vol.4. Vibration machines and processes.) Lavendelis E.E (ed.), Moscow: Mashinostroenie, 1981. 510 p. (In Russian).

[2] V. I. Babitsky Theory of Vibro-Impact Systems and Applications. Berlin: Springer, 1998. 318 p.

[3] Бабицкий В. И., Крупенин В.Л. Машины ударного действия. (The Impact Action Machines). Moscow: Znanie. 1987. 62 p. (In Russian).

[4] Astashev V.K., Babitsky V.I. Ultrasonic Processes and Machines. Dynamics, Control and Applications. Berlin: Springer, 2007. 330 p.

[5] Vladimir Astashev, Vitaly Krupenin About some scientific principles of vibro-impact machine design. Engineering for Rural Development. Jelgava, Latvia, 2018, pp.108-113.

[6] Крупенин В.Л., Мягкохлеб К.Б. Об одном классе авторезонансных машин виброударного действия (On a class of autoresonant machines of vibro-impact action). Доповіді Національної академії наук України, 2014, No. 3, pp 54-59 (In Russian).

[7] Babitsky V.I., Krupenin V.L Vibration of Strongly Nonlinear Discontinuous Systems. Berlin: Springer, 2001. $404 \mathrm{p}$.

[8] Veprik A.M. and other Широкополосные виброударные генераторы механических колебаний (Broadband vibro-impact generators of mechanical oscillations). Leningrad: Mashinostroenie, 1987. 87 p. (In Russian).

[9] Astashev V.K, Babitsky V.I., Kolovsky M.Z. Dynamics and Control of Machines. Berlin: Springer, 2000. $235 \mathrm{p}$.

[10]Вибрации в технике: Справочник. Т.2. Колебания нелинейных механических систем. (Vibration in Engineering: Handbook. Vol.4. Vibration of nonlinear mechanical systems.) Blechman I.I. (ed.), Moscow: Mashinostroenie, 1979. 352 p. (In Russian).

[11] Luo A.C.J., Guo Y. Vibro-impact Dynamics. Berlin: Springer, 2013. 213 p.

[12]Burd V.Sh., Krupenin V.L. Усреднение в квазиконсервативных системах: маятниковые и виброударные системы (Averaging in quasi-conservative systems: pendulum and vibroimpact systems) Moscow: Belyi Veter, 2016. 170 pp.

[13] Andronov A. A., Witt, A. A., Khaikin S. E. Theory of Oscillators. NY: Pergamon Press, 1966.

[14] Babitsky V.I.,Shipilov A.V. Resonant Robotic Systems. Berlin: Springer, 2003. 176 p.

[15] Babitsky V.I., Chitayev M.Y. Adaptive high-speed resonant robot. Mechatronics, 1996, v.6, No. 8, pp. 897-913.

[16] Babitsky V.I. Autoresonant mechatronic systems. Mechatronics, 1995, v.5, No. 5, pp. 483-495.

[17] Akinfiev T.S., Armada, M.A., Fernandez, R. Nondestructive testing of the state of a ship's hull with an underwater robot. Russ. J. Nondestr. Test. 2008, v.44, No.9, pp. 626-633. 